\title{
Characteristics of thermoplastic sugar palm starch/agar blend: thermal, tensile, and physical properties
}

\begin{abstract}
The aim of this work is to study the behavior of biodegradable sugar palm starch (SPS) based thermoplastic containing agar in the range of 10-40 wt $\%$. The thermoplastics were meltmixed and then hot pressed at $140{ }^{\circ} \mathrm{C}$ for $10 \mathrm{~min}$. SEM investigation showed good miscibility between SPS and agar. FT-IR analysis confirmed that SPS and agar were compatible and inter-molecular hydrogen bonds existed between them. Incorporation of agar increased the thermoplastic starch tensile properties (Young's modulus and tensile strength). The thermal stability and moisture uptake increased with increasing agar content. The present work shows that starch-based thermoplastics with $30 \mathrm{wt} \%$ agar content have the highest tensile strength. Higher content of agar (40 wt\%) resulted to more rough cleavage fracture and slight decrease in the tensile strength. In conclusion, the addition of agar improved the thermal and tensile properties of thermoplastic SPS which widened the potential application of this eco-friendly material. The most promising applications for this eco-friendly material are short-life products such as packaging, container, tray, etc.
\end{abstract}

Keyword: Thermoplastic starch; Starch; Agar 